\title{
MOBILTTY PERFORMANCE OF A NEW TRAFFIC DECENTRALIZATION QOS ADMISSION STRATEGY
}

This paper proposes a new Admission Control strategy with Quality of Service (QoS) preservation for GSM networks. System initiated handover calls are used in the central cell in order to decentralize the traffic and redistribute it throughout the whole cluster. Although the channels and the cell size are fixed, the system behaves as if it had the $3 G$ feature called soft capacity. The mobility characteristics of the users are investigated. Low speed and high speed moving users are used as input in the system and the effect of mobility on the proposed algorithm is extracted. Simulation results show that the proposed Admission Strategy is flexible, efficient and very useful in situations when the traffic is centralized.

\section{Introduction}

Resource management is a useful and efficient tool for network optimization. Documentation of several modern network standards contains features like call admission control (CAC), priority and Quality of Service (QoS). The effort is focused on using efficiently the available resources and simultaneously providing a constant and acceptable level of quality to the user. This is usually achieved through network algorithms called CAC algorithms.

In $3 \mathrm{G}$ networks, the resources are easily manipulated throughout the cluster using soft capacity. With the soft capacity feature, the central cell resources are dependent upon the neighbor traffic. Moreover, fractional loading, the utilization of resources becomes even more efficient.

In contrast, Global System for Mobile (GSM) networks have fixed channel resources and cell sizes. The implementation of soft capacity is much more challenging. Soft capacity-like characteristics in GSM networks have been studied in [1] and fractional load techniques have been studied in [2].

Another important issue is the mobility characteristics of the users. In a mobile network, the users are free to roam around the service area, and therefore cause handover traffic. While the handover mechanism is transparent to the user, it is a deciding issue in terms of admission control. It is widely accepted that handover calls are more important than new calls [3] and therefore handovers are provided a sense of priority in admission schemes. The ratio of handover attempts per new calls is called handover ratio This ratio is related to the size of the cells, the mobility characteristics of the users and the admission strategy used.

Several studies have been made on effective CAC algorithms that can be used in GSM networks in order to provide increased QoS. The authors in [4] study guard channel admission control schemes, including new call bounding, cut off priority and new call thinning scheme. In [5] resource allocation and de-allocation is investigated for reducing the blocking probability. A thorough study of dynamic call admission policies is included in [6] and [7], where the algorithms that are presented, are adaptive to the changes of the traffic conditions and the availability of the resources in the cell. In [8] a review of the basic handover schemes that are used in mobile communications is provided. Mobility-based algorithms have been proposed in [9] and [10], where the admission algorithms are handling a call request (new or handover) according to the predictive mobility of the user.

This paper proposes a new way of introducing soft capacity into GSM networks, via system-initiated handovers. The algorithm enables soft capacity by using the unoccupied channels of the neighboring cells and improves the QoS of asymmetrically loaded systems. The performance of the proposed algorithm is tested over several user mobility cases.

\section{Mobility issues}

In order to calculate the handover ratio, the mobility analysis has to be performed. Mobility models have been analysed in [11], [12], [13], [14] and [15]. In [12], [13], [14] and [15], modern mobility models are proposed. However, the disadvantage of these models is the lack of analytical approach. The gamma distribution is found with the procedure of fitting the samples taken from simulations. In this analysis, the interest is focused in urban environments, since centralized traffic conditions usually appear in these cases. As in [11], it is considered that the user is moving in straight lines. The initial location is uniformly random and set $\left(r_{0}, \varphi_{0}\right)$. Then, the user, randomly chooses a destination (declared by $\theta_{i}$ ) and travels with a random velocity (declared by $V_{i}$ ). In case of handover call, the initial position is set on the boundaries of the cell. The four elements have the following probability density functions (pdfs):

\footnotetext{
* Georgios S. Paschos, Efstathios Vagenas, Stavros A. Kotsopoulos

Wireless Telecommunications Lab. - University of Patras, Patras, 26500, Greece, E-mail: kotso@ee.upatras.gr
} 


$$
\begin{aligned}
& f_{\varphi_{0}}(\varphi)=\frac{1}{2 \pi}, 0 \leq \varphi \leq 2 \pi \\
& f_{r_{0}}(r)=\frac{2 r}{R_{e q}}, 0 \leq r \leq R \\
& f_{\theta_{i}}\left(r_{\theta}\right)=\frac{1}{\pi}, 0 \leq \theta \leq \pi
\end{aligned}
$$

where:

$$
\begin{aligned}
& \text { Req }=\sqrt{\frac{3 \sqrt{3}}{2 \pi}} R \\
& f_{V_{i}}(V)=\frac{1}{V_{\max }}, 0 \leq V \leq V_{\max }
\end{aligned}
$$

Using the above statistical results we can estimate the probability density functions pdfs of the resource holding time for a new call $f_{T n}$ and for a handover $f_{T h}$, respectively. $T n$ is the sojourn time of a new call in a cell, and Th the sojourn time of a handover call respectively. In [11], these are calculated and found that follow the following distribution:
The average handover rate is calculated:

$$
\lambda_{H}=\sum_{i=1}^{\infty} \lambda_{N} i P\{H=i]=\lambda_{N} \bar{H}
$$

It is evident that the handover ratio depends only on the user velocity and the cell range up to this point. The results from this analysis will be used in the teletraffic analysis.

\section{QoS Estimation}

The traffic load is assumed largely centralized and handled by the central cell of a seven-cell cluster. Therefore the system, in order to preserve the QoS in the central cell, initiates system handover call requests to the neighboring cells. The CAC scheme searches for available system resources every time a new call or a handover request is issued. If the ongoing traffic exceeds load threshold decided by the provider then the system scans all the established connection in order to find one that can be handed off to a neighboring cell. This decision is called QoS estimation and is based on a QoS Index denoted by QI (QoS Index).

$$
\begin{aligned}
& f_{T n}=\left\{\begin{array}{l}
\frac{8 R_{e q}}{3 V_{\text {max }} \pi t^{2}}\left[1-\sqrt{\left\{1-\left(\frac{t V_{\text {max }}}{2 R_{e q}}\right)^{2}\right\}^{3}}\right], 0 \leq t \leq \frac{2 R_{e q}}{V_{\text {max }}} \\
\frac{8 R_{e q}}{3 V_{\text {max }} \pi t^{2}}, t \geq \frac{2 R_{e q}}{V_{\text {max }}}
\end{array}\right. \\
& f_{T h}=\left\{\begin{array}{l}
\frac{4 R_{e q}}{\pi V_{\text {max }} t^{2}}\left[1-\sqrt{1-\left(\frac{t V_{\text {max }}}{2 R_{e q}}\right)^{2}}\right], 0 \leq t \leq \frac{2 R_{e q}}{V_{\text {max }}} \\
\frac{4 R_{e q}}{\pi V_{\text {max }} t^{2}}, t \geq \frac{2 R_{e q}}{V_{\text {max }}}
\end{array}\right.
\end{aligned}
$$

The channel holding time $T$ is exponentially distributed [11], with mean value $1 / \mu$. The pdf and the cumulative density function (cdf) are:

$$
\begin{aligned}
& f_{T}(t)=\mu e^{-\mu t}, t \geq 0 \\
& F_{T}(t)=1-e^{-\mu t}, t \geq 0
\end{aligned}
$$

We define the probability that a single call requires $k$ handovers $(H=k)$ until it is successfully completed as:

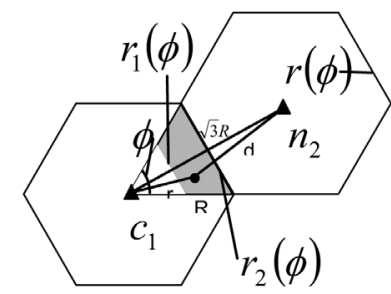

Fig. 1 Representation of parameters for calculating QI.

$$
\begin{aligned}
P[H=k]=P[(T & \left.\left.<T^{n}+k T_{h}\right) \cap\left(T>T_{n}+(k-1) T_{h}\right)\right]=P\left[T<T_{n}+k T_{h}\right] \cdot P\left[T>T_{n}+(k-1) T_{h}\right]= \\
& =\left(1-\int_{0}^{\infty}\left[1-F_{T}(t)\right] f_{T_{n}}+k T_{h}(t) d t\right) \cdot \int_{0}^{\infty}\left[1-F_{T}(t)\right] f_{T_{n}+(k-1) T_{h}}(t) d t= \\
& =\left(1-\int_{0}^{\infty} e^{-\mu t}\left(f_{T_{n}}(t)+k F_{T_{h}}(t)\right) d t\right) \cdot \int_{0}^{\infty} e^{-\mu t}\left(f_{T_{n}}(t)+(k-1) f_{T_{h}}(t)\right) d t
\end{aligned}
$$


Figure 1 shows the case where a mobile station is in a specific action area between the central cell and its neighbor and it receives signals from both base stations. Thus according to QoS estimation the two corresponding signal-to-interference ratios $S I R_{C 1}$ and $S I R_{n 2}$ are compared using $Q I$ as follows:

$$
\begin{aligned}
& S I R_{C_{1}}=\frac{P(r)}{N+I_{a d j}(d)}=\frac{A / r^{2}}{N+I / d^{2}} \\
& S I R_{n_{2}}=\frac{P(d)}{N+I_{a d j}(r)}=\frac{A / d^{2}}{N+1 / r^{2}} \\
& Q I(r)=\frac{S I R_{C_{1}}}{S I R_{n_{2}}}
\end{aligned}
$$

Where $r$ and $d$ represent the distance of the mobile subscriber from the Base Transceiving Stations (BTS) of the central cell and its neighboring cell respectively and $A$ is a factor, constant over $r^{2}$. Additionally, $I$ is the adjacent channel interference and $N$ the background noise. From the above relationship we can derive the $Q I$ in terms of the location of the mobile station in the cell:

$$
Q I=\frac{d^{4}}{r^{4}} \frac{C r^{2}+1}{C d^{2}+1}, \text { where } C=\frac{N}{I}
$$

In the last equation the value of $C$ is assumed known at all times, hence the QoS index is determined by the size and the shape of the action area. Therefore, for a specific threshold value $T h r Q I$, specified by the network provider, we have the following relationship:

$$
\begin{aligned}
& Q I<T h r Q I \rightarrow \text { the call satisfies } Q I \text { criteria } \\
& Q I>T h r Q I \rightarrow \text { the call does not satisfy } Q I \text { criteria }
\end{aligned}
$$

The probability of a user roaming in the action area between the central and a neighboring cell is denoted by $P_{Q I}$. Assuming a load distribution in the cluster of $\lambda(r, \varphi)$ the probability is:

$$
P_{Q I}=1-\frac{\int_{0}^{r_{1}(\phi)} \int_{0}^{\pi / 3} \lambda(r, \phi) d r d \phi}{\int_{0}^{r_{2}(\phi)} \int_{0}^{\pi / 3} \lambda(r, \phi) d r d \phi}
$$

In (4) $r_{1}(\phi)$ is the vector that represents the inside boundary of the action area and $r_{2}(\phi)$ is the vector representation of the boundary of the central cell and its neighboring cell, as shown in figure 1. This probability indicates that a call satisfies the QoS index $Q I$ and is available for system issued handover to a neighboring cell. The QoS estimation is a key function to the proposed call admission strategy and is performed by the QoS estimator, which is a major component of the system model.

\section{Proposed CAC Scheme}

One simple admission strategy is the Handover Reservation algorithm which differentiates between new call and handover call requests and assigns higher priority to the latter by reserving resources for handover calls only, [16, 17]. The algorithm then checks for spare channels. If there are unoccupied channels then the handover request is admitted, otherwise the request is dropped. Similar procedure is followed for new calls, which are admitted if there are unassigned channels available, other than the channels reserved for handover calls or blocked if all channels are occupied. Based on this simple and straight forward CAC scheme, we developed a new $3 \mathrm{G}$-like $\mathrm{CAC}$ algorithm for $2 \mathrm{G}$ networks that guarantees QoS in the cluster. The proposed call admission policy, by means of thresholds controls the traffic decentralization and enables the soft capacity features, despite the fact that the cell sizes and resources in the model are fixed.

In particular, this study assumes a seven-cell cluster system with fixed cell radius. The central cell, which is the cell under study, is assumed to cover an area with excessive traffic while the neighboring cells suffer from much less loading, therefore being used as reservoirs for the excess load of the central cell. This model although theoretical, represents a common situation in real networks. The proposed admission control strategy, based on the above cell-planning model, decentralizes the traffic by handing over the excess load of the central cell to its neighbours. It has to be mentioned that the system initiated handover call requests (sih) are only issued from the central cell towards its neighbors. On the other hand, user initiated handover call requests (uih) due to the mobility of the user, who passes from one cell to another, can equally be issued by all cells in the cluster. The sihs are adequately controlled by the admission scheme in a way that QoS requirements are preserved in the cluster. Figure 2 showcases in a schematic representation, how the admission control strategy achieves traffic decentralization by initiating QoS-driven system handovers.

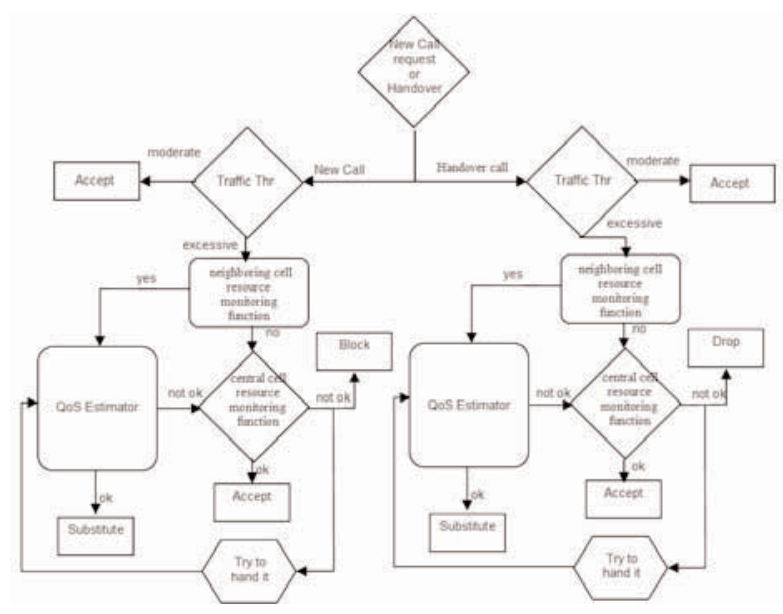

Fig. 2 The schematic diagram of the proposed admission strategy in the central cell.

Main Components of the System

The proposed call admission strategy in figure 2 consists of three major components:

- The neighboring cell resource monitoring function.

- The QoS estimator.

- The central cell resources monitoring function. 
The neighboring cell resource monitoring function checks the resources of the neighboring cell in order to prevent overloading of the neighboring cells. If the neighbor resources are adequate, the QoS estimator is reached, where the QoS index is checked as the last requirement for a sih to be performed. Additionally, the central cell resources monitoring function checks the available channels in the central cell every time that a sih cannot be initiated and the issued call must be serviced within the cell. If this function finds available channels in the central cell then the call is accepted, otherwise it will be handed over to a neighboring cell as a lastditch effort.

\section{The CAC algorithm}

The CAC algorithm is intended to be used in the central cell of a seven cell cluster. For the neighboring cells a resource reservation scheme for handover calls can be assumed, since the system ensures QoS levels in the neighboring cell by means of the resource monitoring function. Thus, only uih call requests are expected in the central cell. The analysis of the algorithm that follows is based on figure 2.

When a new call request is issued, the system checks the number of available resources in the cell. The request will be immediately admitted if the number of used channels $\left(\mathrm{Ch}_{\text {used }}\right)$ is below a threshold value $T h r_{N}$

$$
C h_{\text {used }} \leq T h r_{N} \stackrel{\text { mod erate }}{\longrightarrow} \text { admit new call }
$$

When the available channels have reached the limit value $T h r_{N}$ the algorithm is initiated and the neighboring cell resource monitoring function is called to perform the procedure described above.

$$
\begin{aligned}
& C h_{\text {neigbor }} \leq t h r_{S H} \rightarrow \text { sih anabled } \\
& C h_{\text {neigbor }}>t h r_{S H} \rightarrow \text { sih disabled }
\end{aligned}
$$

Where $t h r_{S H}$ is a threshold for system initiated handovers and in a tolerable central-concerned system we can consider, $t h r_{S H}=N$. When the neighbor cell is in a state less than $t h r_{S H}$, the QoS estimator based on the QoS index $Q I$ either initiates a sih and the new call request is accepted in the central cell or the central cell resources monitoring function is used to allocate the call in the central cell. In the latter case, the new call request is admitted if the spare channels in the new call resource pool are below the threshold, $\operatorname{Res}_{H}$. This value denotes the limit of the new call resource pool.

$$
\begin{aligned}
& \left\{T h r_{N} \leq C h_{\text {used }} \leq \operatorname{Res}_{H}\right\} \cap\{\text { oS ok }] \stackrel{\text { excessive }}{\longrightarrow} \text { substitute } \\
& {\left[T h r_{N} \leq C h_{\text {used }} \leq \operatorname{Res}_{H}\right\} \cap\{\text { ooS not ok }] \stackrel{\text { excessive }}{\longrightarrow} \text { admit }}
\end{aligned}
$$

Furthermore, for a uih request a similar procedure is followed. As long as, the number of occupied channels in the central cell are below the threshold value $T h r_{H}$ then the load in the cell is considered moderate and the request is admitted immediately:

$$
C h_{\text {used }} \leq T h r_{H} \stackrel{\text { mod erate }}{\longrightarrow} \text { uih is admitted }
$$

In a similar way as for new calls, when the traffic is considered excessive, the neighboring cell resource monitoring function is called and (16) enables a sih. Depending on the QoS estimation, the uih either substitutes an ongoing call which in turn is handed over to the neighbor host, or is accepted by the central cell provided the following condition about the available channels is true:

$C h_{\text {used }}<\stackrel{\text { excessive }}{\longrightarrow}$ uih is admitted

Otherwise, the uih request will be blindly handed over to a neighboring cell as a last effort before it is dropped.

\section{Teletraffic Analysis}

In this section the traffic analysis is developed. The assumptions and necessary definitions are firstly stated and then applied in the mathematical analysis that follows.

\section{Assumptions}

i. Cluster traffic normalized to the load variable is assumed as described above.

ii. Poisson traffic with $\lambda$ arrival rates and $\mu$ service rates.

iii. A theoretical seven cells per cluster analysis have been assumed with fixed cell radius $R$.

iv. The neighbor cells surrounding the central cell are assumed symmetrical. This assumption cannot harm the robustness of this analysis since it can be easily modified for a case study that requires asymmetrical cells or traffic.

v. Dirichlet boundaries are assumed and a random neighbor cell is surrounded by four neighbor cells and two central cells.

vi. The background noise is considered greater than the perceived adjacent channel interference when the mobile roams inside the cell which is perfectly reasonable for a typical network with large reuse factor.

vii. We assume a threshold value regarding the QoS estimator which is explained in figure 1 . This graphically presented threshold is yielded from (12).

\section{Definitions}

An important feature for this analysis is the probability that a system initialized handoff request is granted by the QoS estimator, $P_{a c c}$.

$$
P_{\text {acc }}=P\left[\left(Q_{o S} \text { index ok }\right) \cap\left(C h_{\text {neighbor }}\right)\right\}=P_{Q I} \cdot \sum_{n=0}^{t h r S H} P_{n}^{n}
$$

$P_{Q I}$ is the probability for a call to fulfil the QoS index requirements. Furthermore, we define $A_{c}$ as the fact that a new call is either handed onto another cell or a substitute handoff is used as compensation. The result is that the new call will not burden the available cell resources. $B_{c}$ is the respective fact for a handover call case.

The probabilities of $A c$ and $B c$ are defined as $\alpha$ and $b$, and can be used as multipliers of $\lambda$ in the Markov chain.

$$
a=P\left[A_{c}\right\}=P\left[\left(n \geq t h r_{N}\right) \cap(Q o S O k)\right\}=\sum_{n=t h r N}^{N} P_{n}^{c} \cdot P_{a c c}
$$




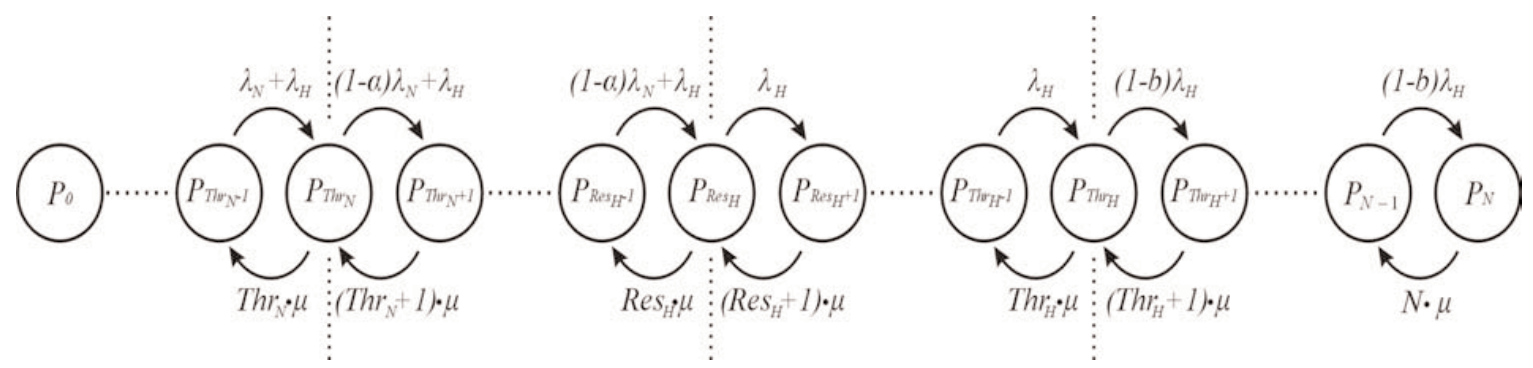

Fig. 3. The Markov chain modified for the needs of the proposed algorithm.

$$
\mathrm{b}=P\left[B_{c}\right]=P\left[\left(n \geq t h r_{N}\right) \cap(Q o S O k)\right\}=\sum_{n=t h r N}^{N} P_{n}^{c} \cdot P_{a c c}
$$

The service rate $\mu$ is considered the same to all cells. The user initiated arrival rates, $\lambda c$ and $\lambda n$ are calculated from the cluster load function using a double integral. The final arrival rates, combining the transactions caused by the algorithm are:

$$
\begin{aligned}
\lambda_{H}^{c} & =\frac{\gamma}{\gamma+1} \lambda^{c}-b \frac{\gamma}{\gamma+1} \lambda^{c} \sum_{n=T h r_{H}}^{N} P_{n}^{c} \\
\lambda_{N}^{c} & =\frac{\gamma}{\gamma+1} \lambda^{c}-a \frac{\gamma}{\gamma+1} \lambda^{c} \sum_{n=T h r_{N}}^{\operatorname{Res}_{H}} P_{n}^{c} \\
\lambda_{H}^{n} & =\frac{\gamma}{\gamma+1} \lambda^{n}+a \lambda_{N}^{c} \sum_{n=T h r_{N}}^{\operatorname{Res}_{H}} P_{n}^{c}+b \lambda_{H}^{c} \sum_{n=T h r_{H}}^{N} P_{n}^{c} \\
\lambda_{N}^{n} & =\frac{1}{\gamma+1} \lambda^{n}
\end{aligned}
$$

Where the indices $c$ and $n$ refer to central and neighbor cell $\gamma$ is the handover ratio which is the same for both neighbor and central cells.

Markov analysis

The classical Birth-Death process is used for the central cell and a representative of the remaining 6 of this one tier analysis. The modifications due to the algorithm are showcased in figure 3 .

The probability that the state $\mathrm{n}$ is occurring in the central cell will be as shown at the bottom of the page (28). It should be noted that apart from $\alpha$ and $b$ the rest comprise a straightforward analysis. However these two percentage variables depend on the load condition of the neighbor cells.

The Markov analysis for a random neighbor cell will differ only by the fact that the iteration algorithm is not used:

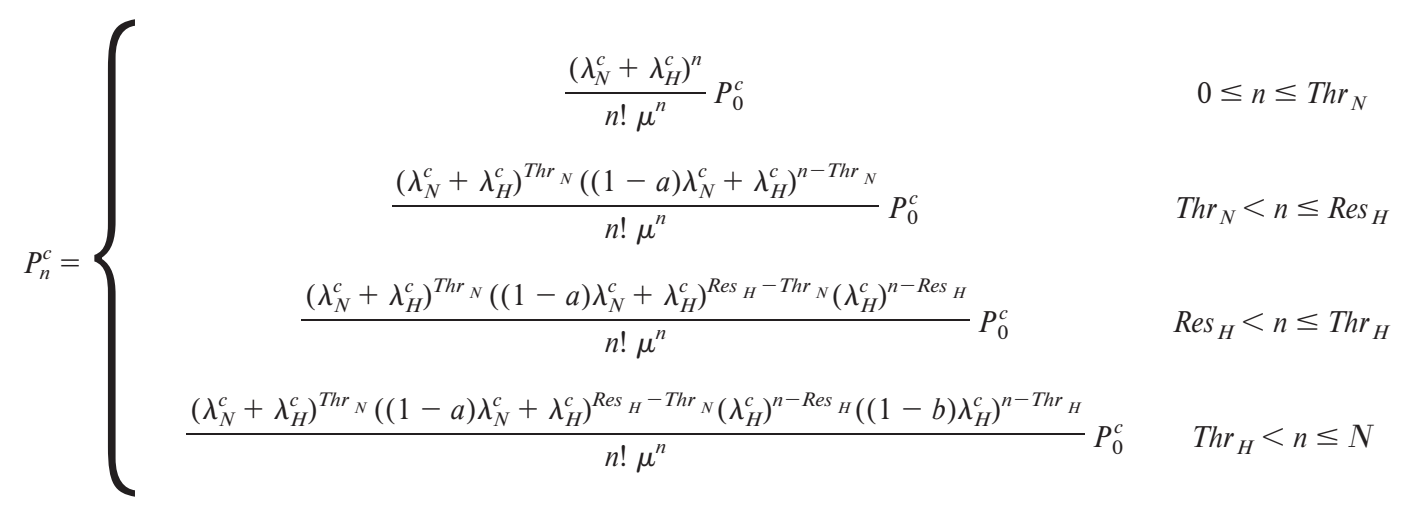

$$
\begin{aligned}
& P_{n}^{c}=1 /\left(1+\sum_{n=1}^{T h r_{N}} \frac{\left(\lambda_{N}^{c}+\lambda_{H}^{c}\right)^{n}}{n ! \mu^{n}}+\sum_{n=\operatorname{Thr}_{N}+1}^{\operatorname{Res}_{H}} \frac{\left(\lambda_{N}^{c}+\lambda_{H}^{c}\right)^{T h r_{N}}\left((1-a) \lambda_{N}^{c}+\lambda_{H}^{c}\right)^{n-T h r_{N}}}{n ! \mu^{n}}+\right. \\
& +\sum_{n=\operatorname{Res}_{H}+1}^{T h r_{H}} \frac{\left(\lambda_{N}^{c}+\lambda_{H}^{c}\right)^{T h r_{N}}\left((1-a) \lambda_{N}^{c}+\lambda_{H}^{c}\right)^{R e{ }_{H}-T h r_{N}}\left(\lambda_{H}^{c}\right)^{n-\operatorname{Res}_{H}}}{n ! \mu^{n}}+ \\
& \left.+\sum_{n=\operatorname{Thr}_{H}+1}^{N} \frac{\left(\lambda_{N}^{c}+\lambda_{H}^{c}\right)^{T h r_{N}}\left((1-a) \lambda_{N}^{c}+\lambda_{H}^{c}\right)^{\operatorname{Res}_{H}-T h r_{N}}\left(\lambda_{H}^{c}\right)^{n-\operatorname{Res}_{H}}\left((1-b) \lambda_{H}^{c}\right)^{n-T h r_{H}}}{n ! \mu^{n}}\right)
\end{aligned}
$$




$$
P_{n}^{n}=\left\{\begin{array}{cc}
\frac{\left(\lambda_{N}^{n}+\lambda_{H}^{n}\right)^{n}}{n ! \mu^{n}} P_{0}^{n} & 0 \leq n \leq \operatorname{Res}_{H} \\
\frac{\left(\lambda_{N}^{n}+\lambda_{H}^{n}\right)^{\text {Res }_{H}}\left(\lambda_{H}^{n}\right)^{n-\operatorname{Res}_{H}}}{n ! \mu^{n}} P_{0}^{n} & \operatorname{Res}_{H}<n \leq N
\end{array}\right.
$$

Where $P_{0}^{n}$ is:

$$
P_{0}^{n}=1 /\left(1+\sum_{n=1}^{\operatorname{Res}_{H}} \frac{\left(\lambda_{N}^{n}+\lambda_{H}^{n}\right)^{n}}{n ! \mu^{n}}+\sum_{n=\operatorname{Res}_{H}+1}^{N} \frac{\left(\lambda_{N}^{n}+\lambda_{H}^{n}\right)^{\operatorname{Res}_{H}}\left(\lambda_{H}^{n}\right)^{n-\operatorname{Res}_{H}}}{n ! \mu^{n}}\right)
$$

The equations 21, 22, 27 and 28 comprise a $2 N+2$ non-linear system of equations and equal unknowns. This system could be apparently large and even larger in case of asymmetrical cells. However numerical analysis methods can be used to efficiently solve the problem.

\section{Iteration method}

In order to solve the above system of equations and extract the valuable simulation results, the iteration method of numerical analysis is utilized [18].

According to this, we make a first estimate of the unknowns $\alpha$ and $b,(\alpha 0, b 0)$. We use this estimate to solve the system and calculate a new set of $\alpha$ and $b$ values. This procedure is called the first iteration. With each new iteration, a new set of $\alpha$ and $b$ values is calculated. A point is then reached when these values are stabilized and no more iterations are needed. The method has converged to the solution of the system. We present the convergence of these two variables for several load values in the following figures 4, 5 .

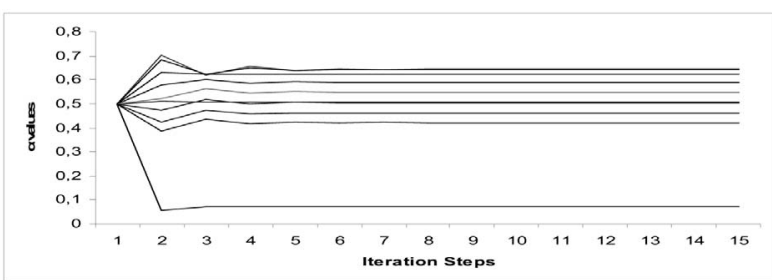

Fig. 4. The convergence of $\alpha$ values for several load cases.

From figures 4,5 , it follows that the method converges rapidly to the solution of the system, within five, at the maximum, itera-

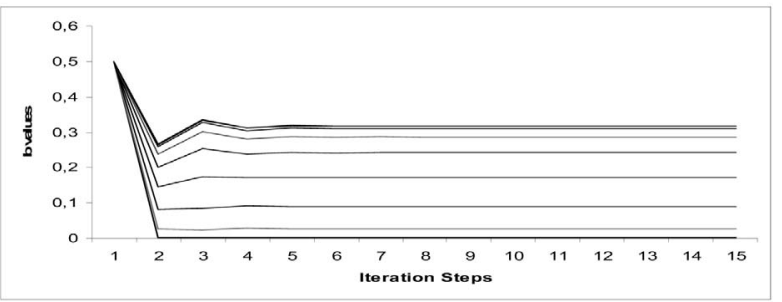

Fig. 5. The convergence of $b$ values for several load cases. tions. Moreover, this happens regardless of the first estimate of values $\left(\alpha_{0}, b_{0}\right)$, or the load situation.

Some measure of instability was encountered in cases of extreme loading where the system oscillates between blocking and non-blocking situation within one step. This happens because of the impact of $\alpha$ and $b$ values incur when the $\lambda / \mu$ is very large. However, this fact cannot affect the rest of our simulation.

\section{Results}

The discussion of the simulation results is based on blocking and dropping probability figures stemmed from the aforementioned analysis. These two indices were chosen as measures because of their ability to grade an admission control algorithm regarding its overall performance.

The proposed system will be tested against the simplified and prevailing Erlang-B and Handover Reservation systems. Such a comparison is fair in this case, since the proposed system is an improved variation of the well-adopted Handover Reservation scheme and the only handicap of the original system is the QoS estimator which is included in the cost of the proposed one. Moreover, the Erlang-B system is always presented as a down boundary for blocking probability and upper boundary of dropping for every scheme in comparison. The traffic is assumed to have circular symmetry $(\lambda(r))$ and the load will be distributed across the radius.

$$
\begin{aligned}
& \lambda(r, \phi)=\left\{\begin{array}{l}
\lambda_{\max }, 0 \leq r \leq R \\
\frac{\lambda_{\max }}{3}, R<r \leq r(\phi)
\end{array}\right. \\
& \lambda_{\max }=\frac{1}{\int_{0}^{\pi / 3} \int_{0}^{r(\phi)} \lambda(r, \phi) d r d \phi}
\end{aligned}
$$

Table 1 contains the rest of simulation inputs. The results are shown in the following figures.

The results for the central cell demonstrate the expected improvement of the system. In both cases (blocking and dropping) the proposed algorithm yields an important gain over the similar 
Simulation values

\begin{tabular}{|c|c|}
\hline & Values \\
\hline Available channels & 36 \\
Average Call Duration & $90 \mathrm{sec}$ \\
$\alpha_{0}, \mathrm{~b}_{0}$ & $0.3,0.3$ \\
Iteration steps & 15 \\
Cell Range & $500 \mathrm{~m}$ \\
High speed mean value & $30 \mathrm{~m} / \mathrm{sec}$ \\
Low speed mean value & $5 \mathrm{~m} / \mathrm{sec}$ \\
\hline
\end{tabular}

Handover Reservation algorithm. This improvement approximates one order of magnitude for heavy load situations. Moreover, the performance of the proposed algorithm challenges even the ErlangB performance in case of blocking with heavy traffic. Figures 8, 9 show the defect of the proposed algorithm. Both probabilities are increased in case of the neighbor cell. However, for a case study of centralized traffic such as this, this deterioration is not of importance. The probabilities are practically held below $10^{-2}$ in any case while the traffic in the central cell is relieved only by the proposed algorithm.

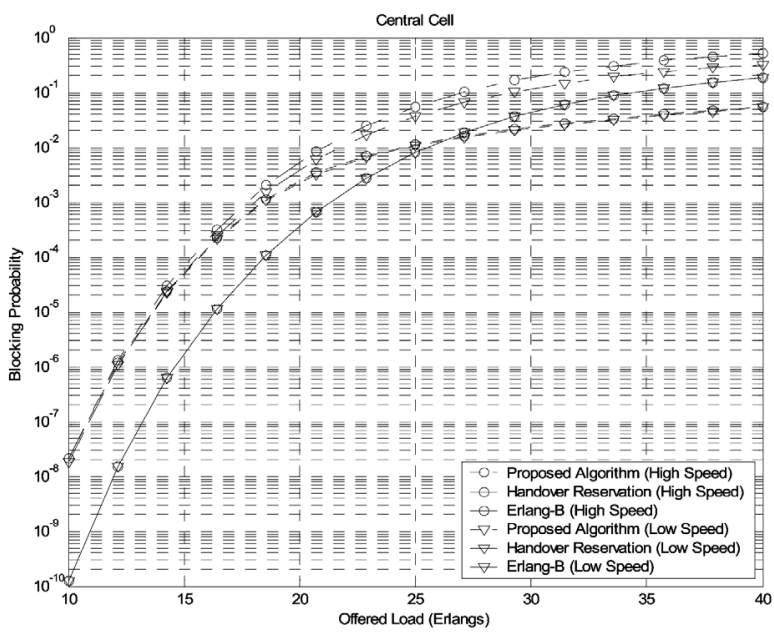

Fig. 6. Blocking Probability for the central cell $\left(t h r_{N}=0.7 \cdot N, \operatorname{Res}_{H}=0.9 \cdot N t_{t h r_{H}}=0.9 \cdot N\right)$

\section{Conclusion}

The implementation of the proposed algorithm concerning the QoS driven admission strategy, can take place on the already installed systems with small interventions. The QoS indicator software structure is necessary along with some custom system messages for support. This can be achieved with the already in-use QoS strategies and measurements regarding the network inherent features. Therefore, the cost of the implementation can be regarded as minor.

Regarding the increased handover traffic, the algorithm possesses a self-defense mechanism. Due to the imposed thresholds,

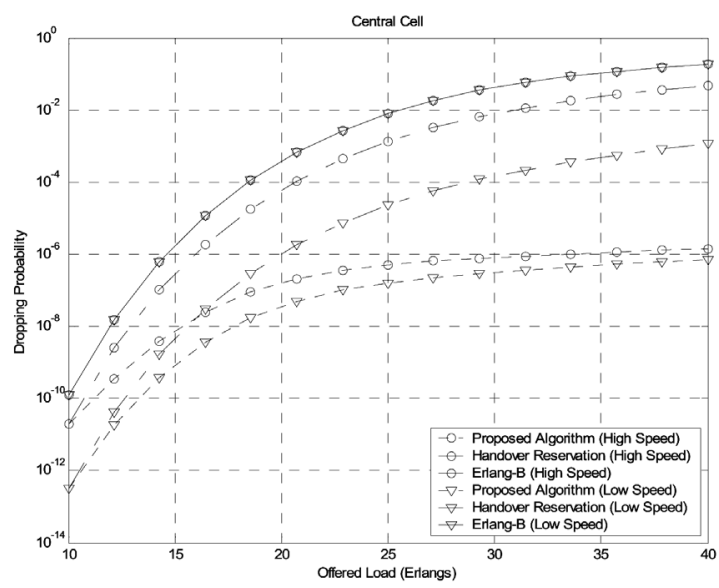

Fig. 7. Dropping Probability for the central cell $\left(t h r_{N}=0.7 . N, \operatorname{Res}_{H}=0.9 . N, t h r_{H}=0.9 . N\right)$

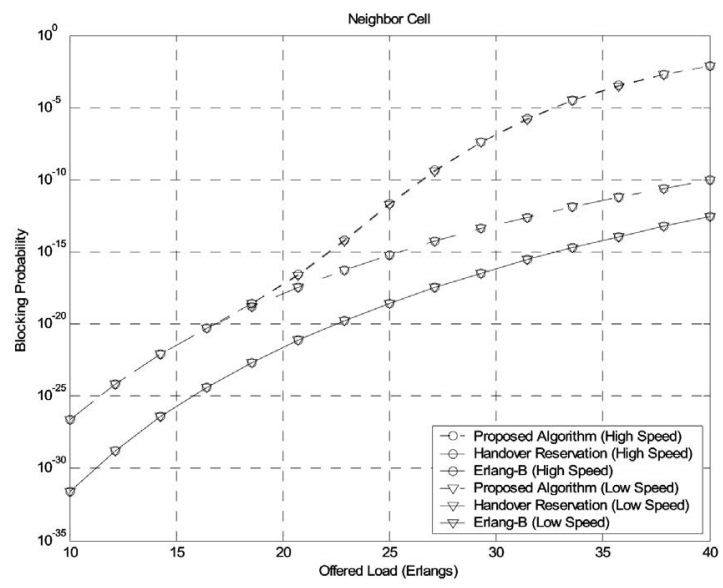

Fig. 8. Blocking Probability for the neighbor cell $\left(t h r_{N}=0.7 . N, \operatorname{Res}_{H}=0.9 . N, \operatorname{thr}_{H}=0.9 . \mathrm{N}\right)$

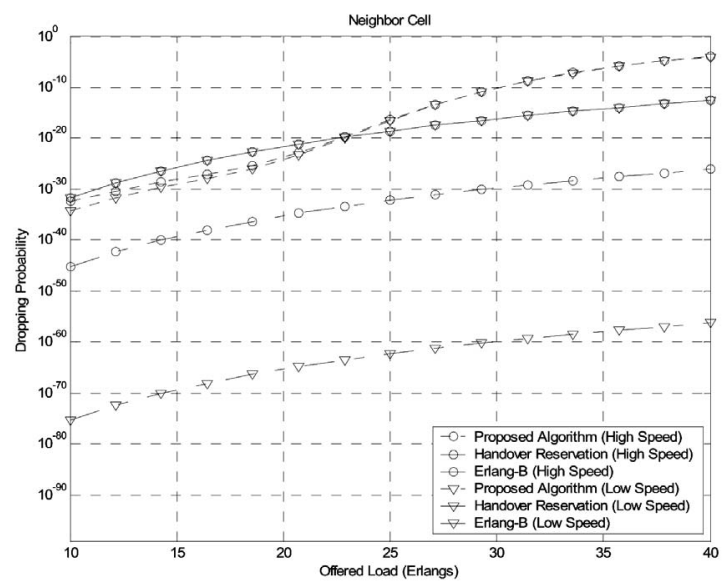

Fig. 9. Dropping Probability for the neighbor cell $\left(t h r_{N}=0.7 . N, \operatorname{Res}_{H}=0.9 \cdot N, t h r_{H}=0.9 \cdot N\right)$ 
the sihs take place only when the system suffers from load peaks and thus, the handover traffic is increased only at times of obvious despair trying to keep the diminishing QoS steady. In addition to that, the handover reservation bandwidth acts as another countering factor and provides further defense against the handover traffic.
The drawn conclusion is that the proposed algorithm creates the soft capacity feature in a $2 \mathrm{G}$ system with fixed cells and resources. Hence it offers a satisfying improvement of QoS for asymmetrically loaded systems by adding an insignificantly small fraction of complexity in the system.

\section{References}

[1] AHMED, M. H., MAHMOUD, S. A.: Soft Capacity Analysis of TDMA Systems with Slow-Frequency Hopping and Multiple-Beam Smart Antennas, IEEE Trans. Veh. Technol., vol. VT-51, No.4, Jul. 2002.

[2] BULDORINI, A., GIUNTINI, E., MAGNANI, N. P.: Performance of a GSM-like system adopting the fractional loading technique, IEEE Wireless Communications and Networking Conference, 1999.

[3] HARDY, W. C.: Measurement and Evaluation of Telecommunications Quality of Service, WILEY 2001.

[4] FANG, Y., ZHANG, Y.: Call Admission Control Schemes and Performance Analysis in Wireless Mobile Networks, IEEE Trans. Veh. Technol., vol. VT-51, Mar 2002.

[5] CHEN, W. Y., WU, J. L., LU, L. L.: Performance Comparison of Dynamic Resource allocation with/without Channel de-allocation in GSM/GPRS Networks, IEEE Commun. Lett., vol. 7, No. 1, Jan. 2003.

[6] WU, S., WONG, K. Y. M., LI, B. O.: A dynamic call admission policy with precision QoS guarantee using stochastic control for mobile wireless networks, IEEE Trans. Network, vol.10, No2, Apr. 2002.

[7] ZHANG, Y., LIU, D.: An adaptive algorithm for call admission control in wireless networks, IEEE Global Telecomm. Confer., GLOBECOM '01, vol.6, 25-29, Nov. 2001.

[8] LOHI, M., BALDO, O., AGHVAMI, A. H.: Handover issues and call admission control in cellular systems, IEEE Veh. Technol. Confer., VTC 1999 - Fall VTS 50th , vol.3, 19-22, Sept. 1999.

[9] HOU, J., FANG, Y.: Mobility-based call admission control schemes for wireless mobile networks, Wireless. Commum. Mob. Comput. 2001, 1:269-282.

[10] ALJADHAI, A. R., ZNATI, T. F.: Predictive Mobility Support for QoS Provisioning in Mobile Wireless Environments, IEEE Journal Select. Areas Commun., vol. 19, No. 10, Oct. 2001.

[11] HONG, D., RAPPAPORT, S. S.: Traffic model and performance analysis of cellular radio telephone systems with prioritized and nonprioritized handoff procedures, IEEE Trans. Veh. Technol., vol. VT-35, Aug. 1986.

[12] CHOI, S. G., CHO, K. R.: Traffic Control Schemes and Performance Analysis of Multimedia Service in Cellular Systems, IEEE Trans. Veh. Technol., vol. 52, No 6, Nov 2003.

[13] BRATANOV, P. I., BONEK, E.: Mobility Model of Vehicle-Borne Terminals in Urban cellular systems, IEEE Trans. Veh. Technol., vol. VT-52, No 4, Jul 2003.

[14] GUERIN, R. A.: Channel occupancy time distribution in a cellular radio system, IEEE Trans. Veh. Technol., vol. VT-35, Aug 1987.

[15] ZONOOZI, M. M., DASSANAYAKE, D.: User mobility modeling and characterization of mobility patterns, IEEE J. on Selected Areas in Comm., 15(7):1239-52, Sept. 1997. 25.

[16] KULAVARATHARASAH, M. D., AGHVAMI, A.H.: Teletraffic performance evaluation of microcellular personal communication networks (PCN's) with prioritized handoff procedures, IEEE Trans. Veh. Technol., vol. VT-48, No.1, pp. 137-152, Jan. 1999.

[17] FANG, Y., ZHANG, Y.: Call Admission Control Schemes and Performance Analysis in Wireless Mobile Networks, IEEE Trans. Veh. Technol., vol. VT-51, Mar 2002.

[18] BURDEN, R. L., FAIRES, J. D.: Numerical Analysis, Brooks Cole, 2000.

[19] WANG, K., LEE, L. S.: Design and analysis of QoS supported frequent handover schemes in microcellular ATM networks, IEEE Trans. Veh. Technol., vol. VT-50, No.4, Jul. 2001. 